\title{
Impact of COVID-19 on People with Mental Disabilities in Japan
}

\author{
Miyako Tazaki
}

Received: 15 June 2020/Accepted: 17 June 2020/Published online: 29 June 2020

(C) Springer Nature India Private Limited 2020

The Japanese government declared on April 16 a nationwide state of emergency amid the corona virus pandemic. Unlike many other countries, however, PCR tests have been given only to patients with severe symptoms of COVID-19. It seems that people who are unable to get tested can become more anxious and fearful than necessary. People also panicked because of the rumors spreading on social media that not only masks and disinfectants but also toilet pepper would disappear from supermarket and convenience store shelves all over Japan.

There are also growing concerns over the so-called "Corona virus Depression"—an economic depression caused by the corona virus outbreak - as Japan's total debt has already exceeded 161.467 million yen. Due to the prolonged self-quarantine, meanwhile, the number of domestic violence and child abuse cases has been drastically increasing. In Japan, no notable demonstrations or riots have been reported following the outbreak of the virus. But people are apparently very frustrated, as illustrated by "quarantine vigilantes" who criticized shops that remained open despite the state of emergency, and anonymous bullying on the

M. Tazaki ( $\square)$

Department of Psychology, Faculty of Medicine, Toho

University, 5-21-16, Omorinishi, Otaku,,

Tokyo 143-8540, Japan

e-mail: mtazaki@med.toho-u.ac.jp
Internet resulting in suicide of a person who was targeted.

Under the circumstances, people with mental disabilities and socially vulnerable people tend to be the first to be dismissed from their jobs. There is a fear of an increase in not only deaths caused by infectious diseases, but also economically and mentally "suicidal deaths at the end of being driven in. People avoid hospitals out of fear of getting infected and refrain from visiting their families at hospitals or day-care facilities. It has resulted in social isolation and the number of people suffering from anxiety disorder has swelled. Washing hands repeatedly has also led to an increase in the number of people being diagnosed with obsessive-compulsive disorder. The conditions among anorexia patients tend to worsen reflecting their anxiety. It is necessary to prevent social isolation among people with mental disabilities as soon as possible by connecting them online with their caretakers.

At facilities for people with disabilities, a large number of residents have lost their lives after being infected with COVID-19, due to a cluster of cases occurring in psychiatric hospitals. This seems to be related to problems that are unique to Japan. At present, the number of patients in medical institutions who suffer from a mental illness exceeds 4 million in Japan. The number of patients admitted to a psychiatric ward is around 280,000, and the number of psychiatric beds available is approximately 340,000; 
this amounts to more than $25 \%$ of the total number of psychiatric beds in the world. Internationally, the average number of hospitalization days for psychiatry is less than 50 days, the shortest stay being one week in Italy and Germany, whereas the average stay in Japan is 390 days. Moreover, since most wards in psychiatric hospitals are still closed in Japan, doors and windows are often closed as a safety measure for patients. This high degree of closure creates an environment in which COVID-19 can easily spread.

According to the NPO Mental Care Council in Tokyo, around 5000 consultations are received per month, and there are claims that people with mental disabilities have been turned away by general hospitals even when they were not displaying any symptoms. It is also claimed they are being denied transfer to a specialized institution for infectious diseases due to their mental illness, even though these patients are elderly with complications such as diabetes, which put them at a high risk of contracting the disease.

COVID-19 reveals the hidden problems of psychiatric patients in our society. It may be high time to radically change how we view mental illnesses. In order to prevent an outbreak occurring in psychiatric hospitals, training on thorough hygiene management and hand washing at facilities should also be introduced to patients immediately. A new law may need to be established to prohibit patients with mental disabilities from receiving proper medical care in the case of emergency. It will take time to completely eliminate discrimination towards people with mental disabilities, but a drastic reduction in the number of long stays of hospitalization would be necessary. This can be achieved by promoting the idea of patients living in a community where they be constantly connected online with their caretakers. In addition, urgent plans and preparation are needed for people with mental disabilities to be able to evacuate safely in a natural disaster such as an earthquake or typhoon, without the risk of being infected with COVID-19. This has not yet been considered and should be dealt with before a crisis arises.

Publisher's Note Springer Nature remains neutral with regard to jurisdictional claims in published maps and institutional affiliations. 